\title{
Stability of Caribbean coral communities quantified by long-term monitoring and autoregression models
}

\author{
Kevin Gross $^{1,3}$ and Peter J. Edmunds ${ }^{2}$ \\ ${ }^{1}$ Biomathematics Program, North Carolina State University, Raleigh, North Carolina 27695 USA \\ ${ }^{2}$ Department of Biology, California State University, Northridge, California 91330 USA
}

\begin{abstract}
Tropical coral reefs exemplify ecosystems imperiled by environmental change. Anticipating the future of reef ecosystems requires understanding how scleractinian corals respond to the multiple environmental disturbances that threaten their survival. We analyzed the stability of coral reefs at three habitats at different depths along the south shore of St. John, U.S. Virgin Islands, using multivariate autoregression (MAR) models and two decades of monitoring data. We quantified several measures of ecosystem stability, including the magnitude of typical stochastic fluctuations, the rate of recovery following disturbance, and the sensitivity of coral cover to hurricanes and elevated sea temperature. Our results show that, even within a $\sim 4 \mathrm{~km}$ shore, coral communities in different habitats display different stability properties, and that the stability of each habitat corresponds with the habitat's known synecology. Two Orbicella-dominated habitats are less prone to annual stochastic fluctuations than coral communities in shallower water, but they recover slowly from disturbance, and one habitat has suffered recent losses in scleractinian cover that will not be quickly reversed. In contrast, a shallower, low-coral-cover habitat is subject to greater stochastic fluctuations, but rebounds more quickly from disturbance and is more robust to hurricanes and seawater warming. In some sense, the shallower community is more stable, although the stability arguably arises from having little coral cover left. Our results sharpen understanding of recent changes in coral communities at these habitats, provide a more detailed understanding of how these habitats may change in future environments, and illustrate how MAR models can be used to assess stability of communities founded upon long-lived species.
\end{abstract}

Key words: autoregression; coral reefs; global climate change; hurricanes; monitoring; seawater temperature; time-series model; U.S. Virgin Islands.

\section{INTRODUCTION}

Nearly every major ecosystem on Earth has been impacted by human activity (Walther et al. 2002, Parmesan 2006). As global climate change intensifies (Stocker et al. 2013) describing and understanding these impacts will remain a primary focus of ecological science (Bellard et al. 2012) and conservation (Parmesan et al. 2013). The principle tool for describing changes in the structure and function of ecosystems is monitoring (Lindenmayer and Likens 2010), which entails the repeated measuring of demographic features or community attributes over time. Monitoring provides a foundation for describing patterns of ecosystem change, but the full value of monitoring can only be realized if it is matched by studies that illuminate the mechanisms driving change (Lovett et al. 2007, Lindenmayer and Likens 2010). Often, a mechanistic understanding of ecosystem change allows those changes to be understood within the rich conceptual framework of ecosystem stability (Holling 1973, May 1974, Ives and Carpenter

Manuscript received 16 May 2014; revised 7 October 2014; accepted 26 November 2014. Corresponding Editor: E. E. Holmes.

${ }^{3}$ E-mail: kevin_gross@ncsu.edu
2007). In turn, the framework of ecosystem stability provides a basis for anticipating the sensitivity of ecosystems to future environmental conditions.

Tropical coral reefs support well-known, diverse, and complex communities that are renowned for their beauty and unique biology, but are also thought to be vulnerable to environmental change (Connell 1978, Nyström and Folke 2001). Over the last 30 years, ecological investigations of coral reefs have been dominated by descriptions of declining abundance of scleractinian corals (Schutte et al. 2010, Jackson et al. 2014). Although there are a few reefs that are still dominated by scleractinians (Sandin et al. 2008), the overall prognosis for the future of coral reefs is poor (Van Hooidonk et al. 2013). Indeed, rising atmospheric $\mathrm{CO}_{2}$ alone may imperil coral reefs through rising seawater temperature (Logan et al. 2014) and declining seawater pH (Kroeker et al. 2013). In addition, scleractinians remain vulnerable to other mortality agents including storms, disease, predators, and algal overgrowth (reviewed in Rosenberg and Loya 2004), each of which may become more severe as environmental change continues. Evaluating the capacity for coral reefs to persist despite this panoply of challenging conditions requires understanding how scleractinians 
respond to multiple stressors. Monitoring offers great promise for this purpose, although studies must extend over sufficiently large scales of space or time to encompass ecologically meaningful variation in environmental conditions. For example, McClanahan et al. (2007) used data from 29 sites over $>1000 \mathrm{~km}$ of the shores of Kenya and the Comoros Islands to resolve the effects of multiple aspects of variation in seawater temperature on coral mortality. In the present study, we use two decades of monitoring data on shallow reefs in St. John, U.S. Virgin Islands, to assess ecosystem stability in response to multiple environmental disturbances.

As monitoring efforts have matured (Gosz et al. 2010), new methods for assessing ecosystem stability from these data have been developed (e.g., Scheffer et al. 2009, Ives and Dakos 2012). Among these, multivariate autoregression (MAR) models (Ives et al. 2003, Hampton et al. 2013) have proven particularly useful for evaluating ecosystem stability, because their parameters connect directly to several measures of stability, including temporal variance, recovery rate following a disturbance, and sensitivity to environmental covariates (Ives et al. 2003, Ives and Carpenter 2007). To date, MAR models have been most commonly applied to communities of short-lived species, such as plankton (Hampton et al. 2013). In the present manuscript, we show how MAR models can be used to assess the effects of acute environmental stressors on coral-reef communities in the Caribbean. While our work was in progress, Cooper et al. (2015) simultaneously and independently developed similar MAR models to forecast the impact of future ocean warming on coral-reef communities on the Great Barrier Reef.

The shallow coral reefs of St. John provide an opportune context to explore how monitoring can be used to assess the stability of reef ecosystems. These reefs have been studied for an extended period with high spatiotemporal resolution (Rogers et al. 2008, Edmunds 2013). Moreover, like most Caribbean reefs (Gardner et al. 2003, Schutte et al. 2010), over the last 28 years, these reefs have endured multiple disturbances including hurricanes (Edmunds and Witman 1991), bleaching, diseases (Miller et al. 2009), and shifts in community composition caused by the die-off of the echinoid Diadema antillarum (Levitan et al. 2014). Recently, Edmunds (2013) described 25 years of benthic community dynamics on the shallow reefs of St. John, and revealed how these communities have changed heterogeneously, and sometimes dramatically, in coral cover and community structure since 1987. Three nearby habitats, two Orbicella-dominated habitats at 9 and 14 $\mathrm{m}$ depth and a near-shore habitat at 7-9 $\mathrm{m}$ depth, displayed different trajectories of change over more than two decades (Edmunds 2013). Spatially heterogeneous trajectories of changing community structure have likely contributed to the kilometer-scale variation that char- acterizes the reefs around both St. John and nearby St. Thomas.

The objectives of this study are to use monitoring data and MAR models to quantify several aspects of the stability of the coral reef communities in St. John, and to use this understanding to evaluate whether the community dynamics of the recent past suggest further deterioration in the future. We focus on stability properties that are related to the so-called stationary distributions of each habitat, which capture the long-run mean and variance in scleractinian coral cover (Ives et al. 2003). Using the stationary distributions, we evaluate four aspects of stability: (1) the (scaled) annual variation in coral cover, (2) the rate at which the community returns to its stationary distribution following disturbance, (3) the sensitivities of mean coral cover to hurricanes and elevated seawater temperature, and (4) the additional temporal trend in mean coral cover over two decades, after accounting for the impact of hurricanes and ocean warming. Together, these four stability properties provide a detailed understanding of how scleractinian corals in St. John are influenced by their environment, and how they are (or are not) likely to change in abundance in the future. A full description of the synecology of these reefs can be found in Edmunds (2002, 2013) and Rogers et al. (2008).

\section{Methods \\ Study site and data collection}

We analyzed the annual benthic community structure of three fringing-reef habitats between Cabritte Horn and White Point along $\sim 4 \mathrm{~km}$ of the south shore of St. John, U.S. Virgin Islands. Field sites and methods are described in Edmunds (2013), and are summarized here, with additional details in Appendix A. Annual monitoring in two habitats, henceforth referred to as Tektite (14 $\mathrm{m}$ depth) and Yawzi Point (9 $\mathrm{m}$ depth), has been conducted since 1987. In both habitats, the reef consists of aggregates of Orbicella annularis with greater coral cover at Tektite (34\% coral cover in 2012, vs. $7 \%$ at Yawzi Point in 2012; Fig. 1, see Plate 1). In 1992, six additional sites between 7 and $9 \mathrm{~m}$ depth were randomly selected on hard substrata, and annual monitoring at these sites has continued since. These sites are treated as random samples of a single habitat that we refer to as the "random-site" (RS) habitat. The benthic community at this RS habitat has been characterized by low $(<5 \%)$ mean coral cover for the duration of the study, although at least 17 scleractinian genera are present. Each of the three habitats was visited annually between May and August, and the benthic community structure described using photoquadrats positioned along permanently marked transects (Appendix A). At all habitats, percent cover of scleractinian corals, macroalgae (generally Halimeda, Lobophora, Padina, and Dictyota), and a combined category consisting of crustose coralline algae, algal turf, and bare space (CTB) were quantified. At the 


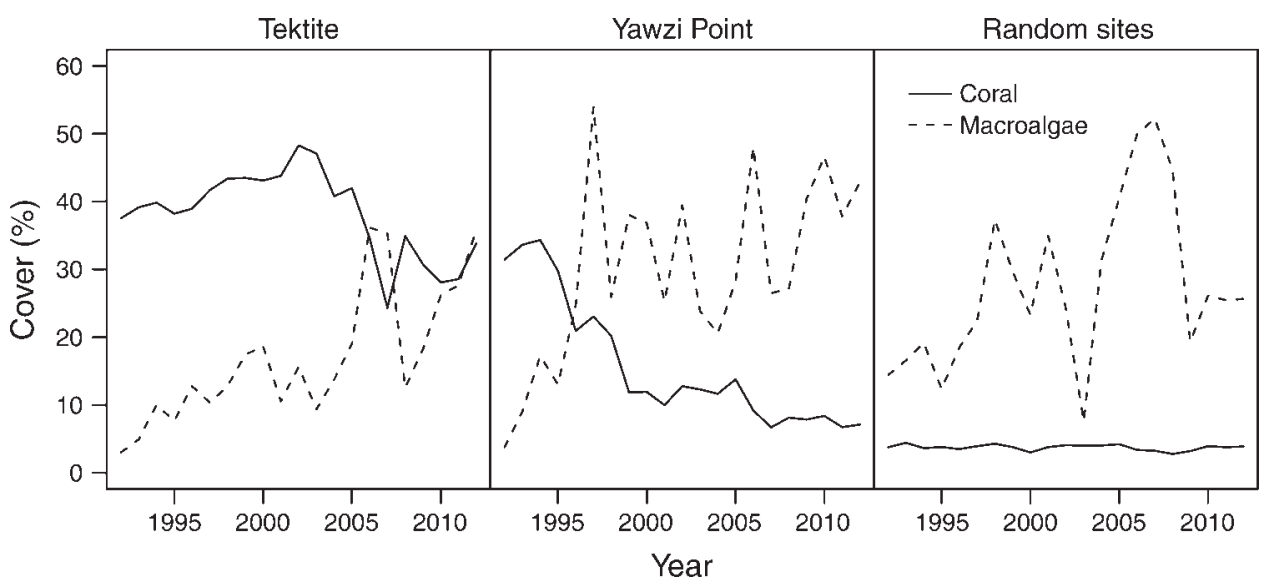

FIG. 1. Average cover of scleractinian corals (solid lines) and macroalgae (broken lines) in three habitats (Tektite, Yawzi Point, and the random-sites habitat) on the south shore of St. John, U.S. Virgin Islands.

RS habitat, greater photographic resolution allowed scleractinians to be separated to genus.

Cover data were averaged across photoquadrats for each habitat (or each site of the RS habitat) and year. For consistency, we analyzed data from 1992-2012 for all three habitats (Fig. 1). Samples were treated as evenly spaced in time, because the planar growth of corals (as detected in photoquadrats) is negligible over a few months. When calculating values for environmental covariates (see below), the interval between samples was assumed to run from 1 August of one year to 31 July of the following year.

Our analysis incorporated covariates for the effects of hurricanes and seawater temperature because these environmental conditions have strong effects on reef corals (Rogers 1993, Lesser 2011), and because they can be quantified for St. John. Hurricane activity was quantified by classifying hurricanes as either major or minor based on their local impact, assigning values of 1 and 0.5 to major- and minor-impact storms, respectively, and then summing over the intervals between surveys. Seawater temperature was measured in situ at 9-14 $\mathrm{m}$ depth using loggers that collected data every $\sim 15$ min (Edmunds and Gray 2014). Temperature was averaged by day and month and converted to degreeheating months (DHMs, after Donner [2009]) using a threshold of $29.3^{\circ} \mathrm{C}$ (Hoegh-Guldberg 1999). DHMs were summed over the intervals between surveys.

\section{MAR modeling}

We analyzed coral reef community structure using a three-category composition consisting of scleractinians, macroalgae, and a third category of "other," which combined CTB with all other substratum cover. CTB was combined with all other cover for modeling because preliminary analyses suggested that distinguishing CTB from other cover did not improve model fit. For the RS habitat, we also completed separate analyses for the most common coral genera: Agaricia, Diploria, Montas- traea (only M. cavernosa), Orbicella, Porites, and Siderastrea (these genera together comprised $90 \%$ of the total coral cover at the RS for these years). We refer to these analyses as the cover analysis and taxonomic analysis, respectively.

Both analyses are based on multivariate autoregressive (MAR) models, and build on the results in Ives et al. (2003) and Cooper et al. (2015). For the present analysis, the MAR model is

$$
\mathbf{x}_{t}=\mathbf{a}+\mathbf{B} \mathbf{x}_{t-1}+\mathbf{C} \mathbf{u}_{t}+\mathbf{z} t+\mathbf{e}_{t} \quad t=2,3, \ldots .
$$

A detailed presentation of the vectors and matrices in Eq. 1 for both the cover and taxonomic analyses appears in Appendix A. For both analyses, abundances are transformed prior to modeling; transformations are described later. In the cover analysis, the vector $\mathbf{x}_{t}$ contains the transformed densities of cover types (e.g., coral, macroalgae, other) at time $t$, while in the taxonomic analysis, $\mathbf{x}_{t}$ contains the transformed densities of coral genera. The vector a contains regression intercepts. Each element in the matrix B quantifies how the abundance of one of the cover types or taxa in $\mathbf{x}_{t}$ affects the per capita population growth rate of either the same (diagonal elements of $\mathbf{B}$ ) or a different (off-diagonal elements of $\mathbf{B}$ ) component of $\mathbf{x}_{t}$ (Ives et al. 2003). The matrix B captures many of the biological processes that are important in structuring these communities, including coral-algal competition (for the cover analysis) and within-genus densitydependence (for the taxonomic analysis). The vector $\mathbf{u}_{t}$ contains the hurricane and sea temperature covariates, and the elements of the matrix $\mathbf{C}$ quantify how those covariates impact the per capita population growth rate of each component of $\mathbf{x}_{t}$. The environmental covariates in $\mathbf{u}_{t}$ are regarded as serially independent draws from a distribution with mean $\boldsymbol{\mu}_{\mathbf{u}}$ and variance matrix $\boldsymbol{\Sigma}_{\mathbf{u}}$. The term $\mathbf{z} t$ captures any (linear) time trend in the components of $\mathbf{x}_{t}$ after accounting for the covariates in $\mathbf{u}_{t}$. (We also enter- 
tained models with quadratic effects of time, but quadratic time effects never provided a statistically significant improvement in fit.) Finally, $\mathbf{e}_{t}$ is a vector of serially independent random errors (independent of $\mathbf{u}_{t}$ ) drawn from a distribution with mean $\mathbf{0}$ and variance matrix $\boldsymbol{\Sigma}_{\mathbf{e}}$. The $\mathbf{e}_{t}$ term captures fluctuations in community composition that arise from processes not explicitly included in the model, including (for example) immigration of larval recruits and fluctuating algal herbivory.

In the typical MAR model, if the spectral radius (the magnitude of the largest eigenvalue) of $\mathbf{B}$ is $<1$, then the distribution of $\mathbf{x}_{t}$ will approach a so-called stationary distribution that captures the long-run mean and variance of the included taxa (Ives et al. 2003, Cooper et al. 2015). Eq. 1 doesn't permit this notion of stationarity, however, because of the term $\mathbf{z} t$. In lieu of a stationary distribution, we will define the quasistationary distribution to be the long-run probability distribution of $\mathbf{x}_{t}$ when the time covariate is fixed at a value $t^{*}$. Throughout, we set $t^{*}$ equal to the value for 2012. We write the mean and variance of this quasistationary distribution as $\boldsymbol{\mu}_{\mathbf{x}}$ and $\boldsymbol{\Sigma}_{\mathbf{x}}$, respectively. (Note that our usage of "quasi-stationary" differs from the typical usage in stochastic-processes theory for a distribution conditioned on non-absorption at a boundary.)

As we show in Appendix B, the mean and variance of the quasi-stationary distribution of $\mathbf{x}_{t}$ are

$$
\begin{gathered}
\boldsymbol{\mu}_{\mathbf{x}}=(\mathbf{I}-\mathbf{B})^{-1}\left(\mathbf{a}+\mathbf{C} \boldsymbol{\mu}_{\mathbf{u}}+\mathbf{z} t^{*}\right) \\
\operatorname{Vec}\left(\boldsymbol{\Sigma}_{\mathbf{x}}\right)=(\mathbf{I}-\mathbf{B} \otimes \mathbf{B})^{-1} \operatorname{Vec}\left(\mathbf{C} \boldsymbol{\Sigma}_{\mathbf{u}} \mathbf{C}^{T}+\boldsymbol{\Sigma}_{\mathbf{e}}\right)
\end{gathered}
$$

(Ives et al. 2003). In Eq. 3, the Vec operator converts a matrix into a vector by stacking the columns of the matrix, and $\otimes$ is the Kronecker product. Eqs. 2 and 3 show how the long-run distribution of the taxa in $\mathbf{x}_{t}$ depends on interactions among taxa (B), the environment $\left(\boldsymbol{\mu}_{\mathbf{u}}\right.$ and $\boldsymbol{\Sigma}_{\mathbf{u}}$ ), how taxa respond to the environment $(\mathbf{C})$, and time $(\mathbf{z})$.

Eqs. 2 and 3 give rise to several different measures of stability (Ives et al. 2003). First, the coefficient of variation $(\mathrm{CV})$ of coral cover at the quasi-stationary distribution provides a scaled measure of annual variation in abundance that is comparable across ecosystems (May 1974). Second, the spectral radius of B quantifies how quickly the ecosystem returns to its quasi-stationary distribution following a disturbance (Ives et al. 2003). When the spectral radius is small, the ecosystem returns to its quasi-stationary distribution more quickly than if it is large. In contrast to the CV, the spectral radius is a property of the entire community.

We can quantify how the community's quasi-stationary distribution depends on the environment or on time by differentiating Eqs. 2 and 3. For example, the dependence of the mean abundance of the taxa in $\mathbf{x}_{t}$ on the environment or on time is given by

$$
\frac{d \boldsymbol{\mu}_{\mathbf{x}}}{d \boldsymbol{\mu}_{\mathbf{u}}}=(\mathbf{I}-\mathbf{B})^{-1} \mathbf{C}, \quad \frac{d \boldsymbol{\mu}_{\mathbf{x}}}{d t^{*}}=(\mathbf{I}-\mathbf{B})^{-1} \mathbf{z} .
$$

Similarly, the dependence of the variance of the taxa in $\mathbf{x}_{t}$ on the variance of the (random) environmental covariates is found by

$$
\frac{d \operatorname{Vec}\left(\boldsymbol{\Sigma}_{\mathbf{x}}\right)}{d \operatorname{Vec}\left(\boldsymbol{\Sigma}_{\mathbf{u}}\right)}=(\mathbf{I}-\mathbf{B} \otimes \mathbf{B})^{-1}(\mathbf{C} \otimes \mathbf{C}) .
$$

We refer to $d \boldsymbol{\mu}_{\mathbf{x}} / d \boldsymbol{\mu}_{\mathbf{u}}$ and $d \operatorname{Vec}\left(\boldsymbol{\Sigma}_{\mathbf{x}}\right) / d \operatorname{Vec}\left(\boldsymbol{\Sigma}_{\mathbf{u}}\right)$ as "sensitivities," and to $d \boldsymbol{\mu}_{\mathbf{x}} / d t^{*}$ as the "trend". Eqs. 4 and 5 capture how interactions among cover types or taxa in the reef community (as quantified in B) buffer the direct impacts of environment $(\mathbf{C})$ or time $(\mathbf{z})$ to determine the long-run community composition.

\section{Special considerations for data from St. John}

Consideration 1: Data transformations.-Cover data are collected as compositions, which violate the constant variance assumption of the MAR errors. Here, we follow Cooper et al. (2015) by transforming composition data to a more suitable scale using an isometric log-ratio (ilr) transformation (Egozcue and Pawlowsky-Glahn 2011; details in Appendix A). A linear approximation is used to convert results back to the proportion scale for reporting. Throughout, we use the notation $\mathbf{x}$ to indicate population densities on the transformed scale, and the notation $\mathbf{p}$ to indicate population densities on the proportion scale. Because the ilr scale is based on a $\log$ transformation, and because the MAR model is linear on the transformed scale, the effects of environmental covariates on reef community composition are multiplicative on the proportion scale. Thus, we report sensitivities as the proportional change in the mean cover with respect to the environmental covariates (i.e., $d \ln \boldsymbol{\mu}_{\mathbf{p}} / d \boldsymbol{\mu}_{\mathbf{u}}$, or equivalently $\left(d \boldsymbol{\mu}_{\mathbf{p}} / d \boldsymbol{\mu}_{\mathbf{u}}\right) \times\left(1 / \boldsymbol{\mu}_{\mathbf{p}}\right)$, instead of $d \boldsymbol{\mu}_{\mathbf{p}} / d \boldsymbol{\mu}_{\mathbf{u}}$; Appendix A). At the RS, summed coral cover never exceeded $10 \%$, and thus data for coral genera were log-transformed to stabilize the variance. Prior to transformation, one-half of the smallest non-zero observation in the data set was added to each data point to accommodate years when a genus was not observed at a particular site (as occurred at $18 \%$ of all genus $\times$ year $\times$ site combinations).

Consideration 2: Data from multiple locations.-For the RS data, we assume that the mean abundance of the cover types and coral genera differed across the sites, but that the interactions among taxa, the effect of the environment, and the distribution of the environmental covariates were the same across sites. Based on an exploratory data analysis, we assumed a common time trend across sites for the cover analysis, but site-specific time trends for the taxonomic analysis. Thus, the MAR model included a site-specific intercept vector a (and a site-specific trend vector $\mathbf{z}$ in the taxonomic analysis), but all other model parameters were common across sites. For analysis and reporting, we averaged site- 


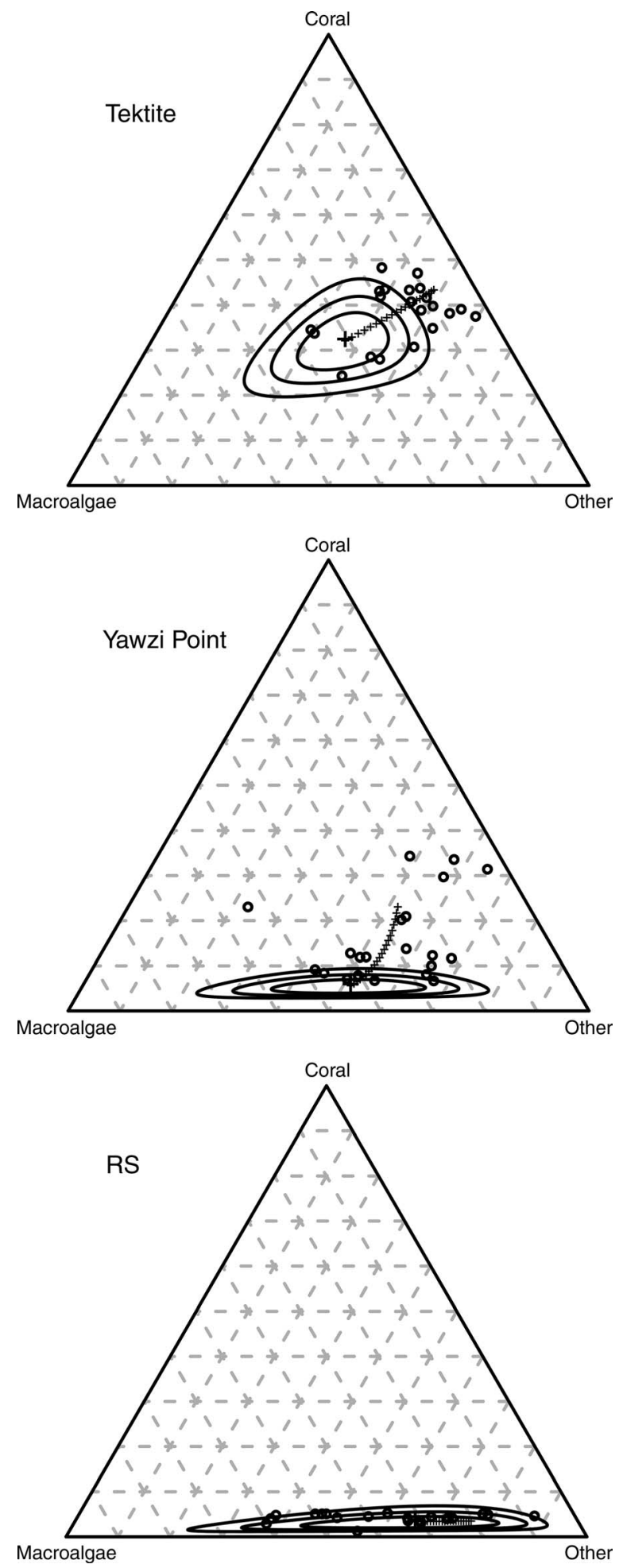

FIG. 2. Cover composition of coral, macroalgae, and other cover for three shallow habitats on the south shore of St. John. Large plus (+) symbols show the metric center (Appendix A) of the quasi-stationary distribution for 2012, and are enclosed in approximate $50 \%, 80 \%$, and $95 \%$ probability contours. Approximate probability contours require an assumption that environmental covariates in $\mathbf{u}_{t}$ and model residuals in $\mathbf{e}_{t}$ are specific parameters across sites to yield a common quasidistribution that describes a typical site.

Consideration 3: Competition among coral genera at low cover sites.-Coral cover at the RS habitat is sufficiently low that coral colonies rarely encounter one another on the benthos, and thus competition among coral genera is unlikely to be important. In fact, a model with interactions among genera only provided a marginally improved fit compared to a model without those interactions $\left(F_{30,600}=1.46, P=0.055\right.$; this test is approximate, because it requires an assumption that the $\mathbf{e}_{t}$ are normally distributed). Thus, we set the offdiagonal elements of $\mathbf{B}$ to 0 in the taxonomic model.

\section{Parameter estimation and statistical inference}

MAR model parameters were estimated with conditional least-squares (Ives et al. 2003). Standard regression diagnostics were used to evaluate the quality of the model fits and homogeneity of variance (Appendix C). To accommodate non-normality of residuals, a nonparametric bootstrap with 5000 bootstrap replicates was used for statistical inference (Ives et al. 2003). Bootstrap replicates that generated a non-stationary model (spectral radius $>1 ;<0.1 \%$ of bootstrap replicates) were discarded. Bootstrap distributions for a few parameter estimates were strongly skewed, so a robust bootstrap standard error with $\alpha=0.95$ (Efron and Tibshirani 1994) was used throughout. For the RS data, residuals were re-sampled as yearly blocks to preserve any spatial correlation among the sites. We conducted a small simulation study (Appendix D) to investigate the statistical properties of our estimators.

\section{RESUlts}

\section{Cover at three habitats}

In the estimated quasi-stationary distribution for 2012 (Fig. 2), coral cover is higher at Tektite $(31.7 \% \pm 4.1 \%$ at the distribution's metric center [mean $\pm \mathrm{SE}$ ]; Appendix A) and low at both Yawzi Point (5.3\% \pm $0.8 \%)$ and the RS $(3.0 \% \pm 0.9 \%)$. Macroalgae cover was lowest at Tektite $(30.9 \% \pm 3.8 \%)$, highest at Yawzi Point $(43.1 \% \pm 6.7 \%)$ and intermediate at the RS $(32.2 \% \pm$ $8.3 \%$ ). At all three habitats, the center of the quasistationary distribution has shifted toward greater algal cover from 1992-2012, although the extent to which algal cover has increased at the expense of decreases in coral cover (vs. decreases in other) differs across the three habitats (Fig. 2).

normally distributed. See Appendix D for a comparison to the quasi-stationary distribution when these normality assumptions are relaxed. Small plus symbols trace how the center has changed from 1992 to 2012. Open circles show annual compositions. Data shown for the random-sites [RS] habitat are from site RS-15 (see map in Edmunds [2013]), which had the third greatest annual coral cover of the six sites. Other sites are shown in Appendix A: Fig. A1. 

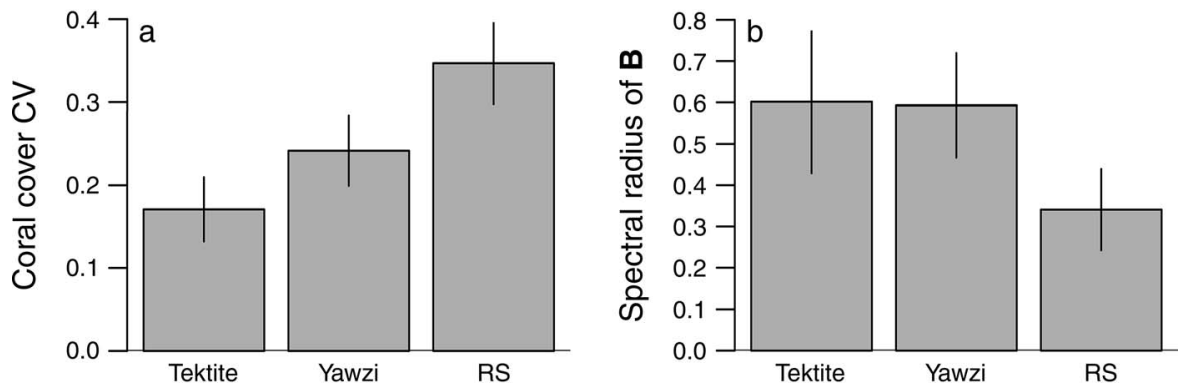

FIG. 3. Two measures of stability of benthic communities at Tektite, Yawzi Point, and random-site (RS) habitats. (a) Coefficient of variation of coral cover in the quasi-stationary distribution. (b) Spectral radius of the matrix of interaction coefficients B. Larger values of the spectral radius indicate slower return to the quasi-stationary distribution following a disturbance. Error bars are \pm 1 robust bootstrap SE.

$\mathrm{CV}$ of coral cover and the spectral radius quantify the stability of each habitat at the quasi-stationary distribution (Fig. 3). The estimated CV of coral cover was lowest at Tektite, intermediate at Yawzi Point, and largest at the RS (Fig. 3a). In contrast, the spectral radii at Tektite and Yawzi Point were roughly equal, but larger than the spectral radius at the RS (Fig. 3b). The estimated spectral radii suggest that communities at the RS habitats return to a pre-disturbance state more quickly than communities at either Tektite or Yawzi Point. Using conventional thresholds for statistical significance, the $\mathrm{CV}$ of coral cover at Tektite is significantly less than the $\mathrm{CV}$ of coral cover at the RS (two-tailed $P=0.006$ ). No other pairwise comparison between habitats is significant at the $5 \%$ level for either $\mathrm{CV}$ or spectral radius. Our simulation study (Appendix D) suggested that there may be considerable bias (on the order of 20-30\%) in the estimate of spectral radius for time series of this length. Bias of this magnitude suggests that comparisons of estimated spectral radii should be interpreted cautiously.
Sensitivities (Eq. 4) of coral cover quantify how hurricanes and seawater temperature impact community composition at these habitats (Fig. 4a and b). Fig. 4a and $\mathrm{b}$ show proportional sensitivities of coral cover calculated with respect to a $50 \%$ increase in hurricane impact and to a $50 \%$ increase in DHMs. For example, the proportional sensitivity of $-5.5 \% \pm 2.5 \%$ to hurricane activity for Yawzi Point suggests that a 50\% increase in hurricane activity is associated with a 5.5\% proportional decrease in long-term coral cover. In contrast, at Tektite, the same change in hurricane activity is associated with a $0.2 \% \pm 1.6 \%$ decrease in coral cover. Estimated annual trends (Fig. 4c) show how coral cover has changed at each habitat after accounting for the impacts of hurricanes and sea temperature. Using traditional thresholds of statistical significance, but without correcting for multiple comparisons, the following sensitivities and trends are statistically distinguishable from zero: the sensitivity of coral cover at Yawzi Point to hurricane activity $(P=0.008)$ and temperature $(P=0.002)$, and the trend in coral cover at

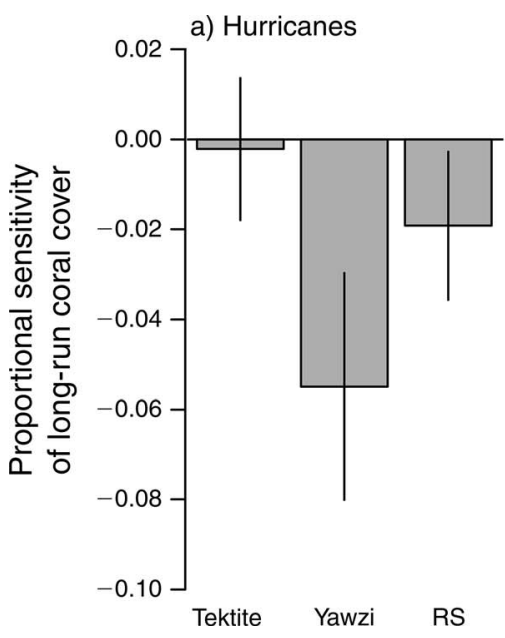

b) Temperature

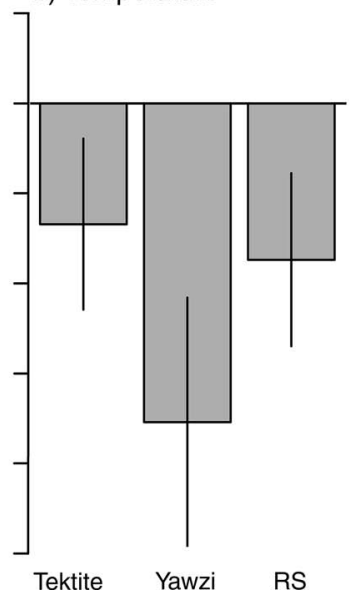

c) Additional trend

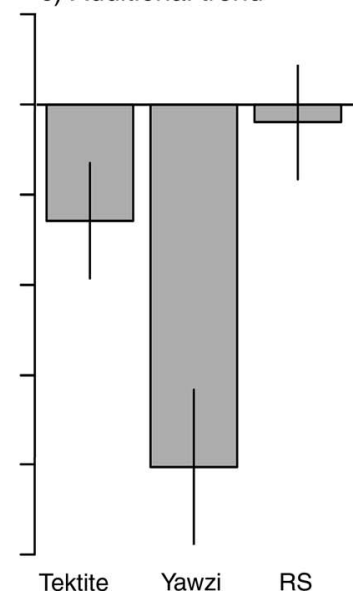

FIG. 4. Proportional sensitivities (i.e., $\left(d \boldsymbol{\mu}_{\mathbf{p}} / d \boldsymbol{\mu}_{\mathbf{u}}\right) \times\left(1 / \boldsymbol{\mu}_{\mathbf{p}}\right)$, where $\boldsymbol{\mu}_{\mathbf{p}}$ gives the mean cover composition and $\boldsymbol{\mu}_{\mathbf{u}}$ gives the mean of the environmental covariates) and annual trend (i.e., $\left.\left(d \boldsymbol{\mu}_{\mathrm{p}} / d t^{*}\right) \times\left(1 / \boldsymbol{\mu}_{\mathrm{p}}\right)\right)$ of mean coral cover at the quasi-stationary distribution for Tektite, Yawzi Point, and the random sites (RS). Sensitivities are calculated with respect to (a) a $50 \%$ increase in annual hurricane activity, (b) a 50\% increase in degree-heating months (DHM) per year, and (c) the additional annual trend, after accounting for hurricanes and sea temperature. Error bars are \pm 1 robust bootstrap SE. 


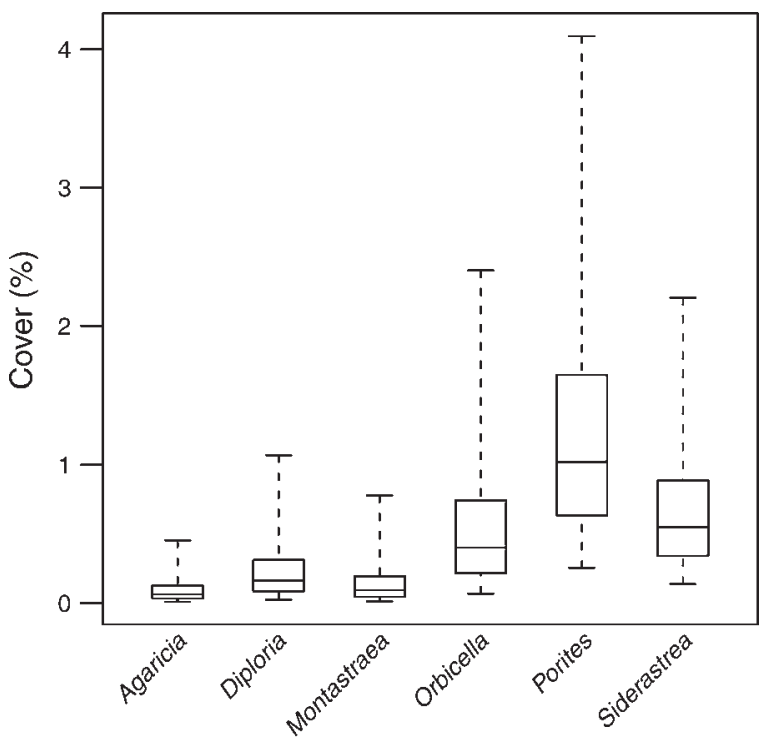

FIG. 5. Quasi-stationary distributions of six coral genera found at the random-sites habitat. For each genus, solid lines indicate medians, the boxes extend from the lower to the upper quartiles, and whiskers extend from the 2.5th to the 97.5th percentiles.

Tektite $(P=0.028)$ and Yawzi Point $(P=0.003)$. Additionally, the trend at Yawzi Point is significantly different from the trend at Tektite $(P=0.024)$ and at the RS $(P=0.003)$. Sensitivities and trends computed for macroalgae (Appendix E) suggest that seawater warming will increase macroalgal cover at all three habitats, while hurricane activity will have a more pronounced impact on macroalgal cover at Yawzi Point than at either Tektite or the RS habitat. Sensitivities of the variance in coral cover (Eq. 5) suggest that variability in hurricanes and seawater temperature has a greater impact on annual variability in coral cover at Yawzi Point than at the other two habitats (Appendix E), although the statistical uncertainty in these sensitivities is large.

\section{Coral genera at the RS habitat}

Quasi-stationary distributions for the 6 common coral genera in the RS were strongly right skewed (Fig. 5). The strong skew of the quasi-stationary distribution suggests that these genera will occasionally occur at relatively high abundance, but will be relatively scarcer during the majority of years and at the majority of sites. The genus with the greatest predicted abundance is Porites, which is predicted to cover $1.31 \% \pm 0.14 \%$ of the benthos under stationary conditions, which equates to $42 \%$ of the total cover of these six most common genera at the RS.

Estimated sensitivities of coral genera suggest that Agaricia is sensitive to both hurricanes (Fig. 6a; $P=$ 0.018 ) and seawater temperature (Fig. 6b; $P<0.001$ ). None of the other genera show statistically significant sensitivities to either environmental covariate at the RS habitat. After accounting for hurricanes and sea temperature, Diploria $(+6.8 \% \pm 1.8 \%$ per yr, $P<$ $0.001)$ and Porites $(+7.0 \% \pm 0.6 \%$ per yr, $P<0.001)$ show evidence of increasing cover over the duration of this study (Fig. 6c). While these two rates are comparable, they reflect different patterns of growth,
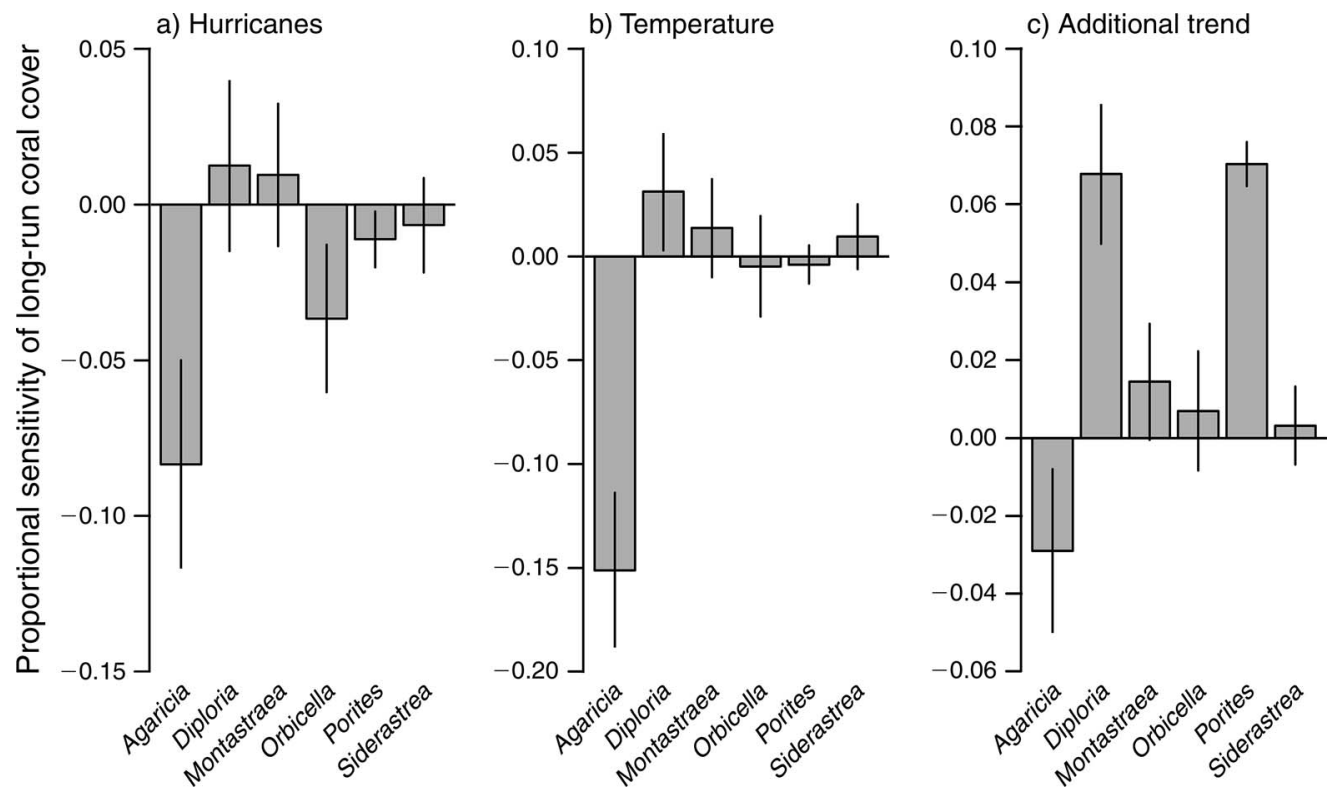

FIG. 6. Proportional sensitivities (i.e., $\left.\left(d \boldsymbol{\mu}_{\mathbf{p}} / d \boldsymbol{\mu}_{\mathbf{u}}\right) \times\left(1 / \boldsymbol{\mu}_{\mathbf{p}}\right)\right)$ and annual trend (i.e., $\left.\left(d \boldsymbol{\mu}_{\mathbf{p}} / d t^{*}\right) \times\left(1 / \boldsymbol{\mu}_{\mathbf{p}}\right)\right)$ of mean coral cover for six coral genera found at the random-sites habitat. Sensitivities are calculated with respect to (a) a $50 \%$ increase in annual hurricane activity, (b) a $50 \%$ increase in DHM per year, and (c) the additional annual trend, after accounting for hurricanes and sea temperature. Error bars are \pm 1 robust bootstrap SE. 
as Diploria has increased from near absence in 1992 to low abundance in 2012, while Porites has increased from moderate to (relatively) high abundance (Fig. 5). None of the other genera showed a statistically significant trend over time.

Estimates and robust bootstrap standard errors of MAR model parameters are presented in Appendix E.

\section{Discussion}

Anticipating the responses of ecosystems to future environmental change is one of the preeminent challenges facing contemporary ecology. On tropical coral reefs, large recent declines in coral abundance together with a multitude of environmental threats to scleractinian fitness have led to gloomy forecasts for the fate of reefs (Hoegh-Guldberg et al. 2007, Van Hooidonk et al. 2013). Nevertheless, our understanding of how coral reef communities respond to environmental change is still spatially, temporally, and taxonomically coarse. Resolving how scleractinians are impacted by separate environmental stressors is necessary to acquire a deeper understanding the natural variation in coral dynamics on reefs. Here, we analyzed two decades of dynamics of coral reef communities in St. John to obtain a more detailed understanding of how these communities respond to environmental change. The four stability metrics that we calculated provide both a sharper retrospective understanding of the drivers of recent community shifts, and enable predictions of how these communities may continue to change in the near future. Further, we suggest below that the stability of these communities can be connected to their known synecology, as it has been described elsewhere (e.g., Edmunds 2002, 2013, Rogers et al. 2008). This connection to the processes that govern community dynamics, structure and composition is important because it emphasizes that stability is an emergent property of those processes. In turn, this mechanistic understanding suggests how this study of corals in St. John may enlighten the study of other coral communities with similar structure and taxa.

In the Orbicella-dominated habitats at Tektite and Yawzi Point, as elsewhere in the Caribbean (Jackson et al. 2014), coral cover has declined in recent decades, and macroalgal cover has increased concomitantly. Our estimate of the quasi-stationary distribution (depicted by the approximate probability contours in Fig. 2) suggests that these shifts in community composition are not merely random walks resulting from typical annual stochastic fluctuations, but instead are evidence that the communities have responded in a directional manner to sustained alterations of their environment. Although both Tektite and Yawzi Point habitats exhibit qualitatively similar trends in cover composition, the magnitude of those trends differs, with more severe coral-cover decline at Yawzi Point. The CV and spectral radii of the Tektite and Yawzi Point habitats are also similar (Fig. 3), suggesting that both habitats ultimately display similar variability (after adjusting for the several-fold differences in recent coral cover) and recovery rates from disturbance. The immediate impacts of these disturbances may be very different, however. Sensitivity calculations suggest that corals at Yawzi Point are vulnerable to hurricanes, while corals at Tektite are considerably less afflicted by these storms (Fig. 4a). This difference is likely related to the protection from damaging storm waves provided by Tektite's greater depth and position in the lee of Cabritte Horn (Edmunds and Witman 1991, Edmunds 2013). Seawater warming also reduced coral cover at Yawzi Point (Fig. 4b), but had a more mild (and statistically insignificant) impact on coral cover at Tektite.

The less severe effect of temperature on coral at Tektite is not consistent with a previous report of bleaching, coral disease, and a striking decline in coral cover at Tektite in 2005 in the wake of an unusually warm summer (Miller et al. 2009). While we observed a modest $(17 \%)$ decline in coral cover at Tektite from 2005 to 2006 , we observed a more severe $(30 \%)$ decline the following year, when seas were cooler (but still eclipsed the $29.3^{\circ} \mathrm{C}$ bleaching threshold). The difference between our findings and those of Miller et al. (2009) may illustrate heterogeneous responses to seawater warming over spatial scales as small as hundreds of meters. Or, it may also suggest that the full impact of thermal stress on coral health may take multiple years to manifest as reduced cover. Delayed impacts of thermal stress could be generated by the slow onset of disease, by mortality that accrues only after consecutive years of compromised coral performance (Knowlton et al. 1990), or by the splitting of large colonies into small ones that subsequently experience greater mortality (HernándezPacheco et al. 2011). If delayed impacts of disturbance are important, then the full impact of variation in seawater temperature on coral cover may be greater than our results suggest, because the MAR model (at least with a single time lag) only captures the immediate (i.e., same-year) impacts of environmental disturbances. Although beyond the scope of the present study, MAR models with multiple time lags may provide a profitable avenue for exploring this possibility.

Delayed impacts of disturbance may also contribute to the additional decrease in coral cover found at both Tektite and Yawzi Point after accounting for the immediate impacts of hurricanes and seawater warming (Fig. 4c). Other factors that might have contributed to this additional coral loss include declining seawater $\mathrm{pH}$ (Gledhill et al. 2008), low post-settlement success of coral recruits (Arnold et al. 2010), and dynamic feedback in which decreases in reef structural complexity reduce algal herbivory (Mumby et al. 2007).

The stability of the low-coral cover communities at the RS habitat differs from Tektite and Yawzi Point. When scaled relative to abundance, annual coral cover at the RS is highly variable, both for all scleractinians (Fig. 3a), and for individual genera (Fig. 5). The spectral 


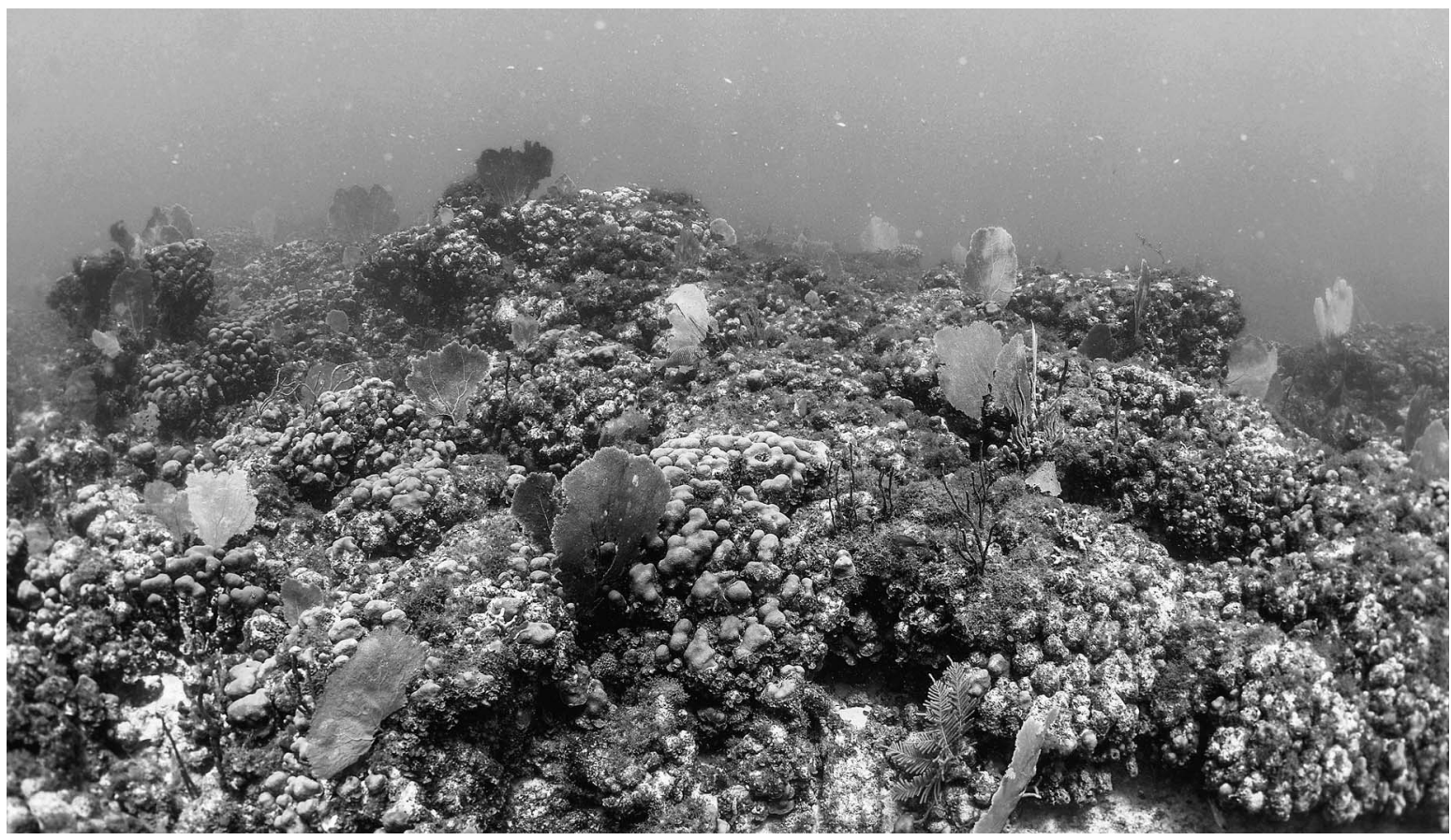

Plate 1.. Shallow reef at $8 \mathrm{~m}$ depth off Yawzi Point, St. John, U.S. Virgin Islands. This image, taken in 2012, shows a reef dominated by a framework of Orbicella annularis, much of which has died back to small patches of tissue on the tops of columnar colonies. Macroalgae profusely grow on this framework. Our Yawzi Point study site is located in this habitat, and the two other habitats are found on adjacent areas of reef. Photo credit: P. J. Edmunds.

radius of the RS suggests that this habitat recovers quickly from disturbance, although differences in spectral radii among the three habitats are not statistically significant (Fig. 3b). Coral cover at the RS habitat appears somewhat vulnerable to ocean warming, but relatively robust to hurricanes, and (unlike corals at Tektite or Yawzi Point) shows no evidence of an additional temporal trend in abundance (Fig. 4). Taken together, these stability properties are consistent with our previous interpretation (Edmunds 2013) that the RS habitat is characterized by rapid population turnover of scleractinians, regular replenishment of coral populations through external larval recruitment, and a relative insensitivity to environmental factors threatening coral reefs.

With respect to the coral genera at the RS habitat, our results support the suggestion that the eurytopic genus Porites will proliferate on benthic communities on shallow reefs in the Caribbean in near future (Burman et al. 2012, Darling et al. 2012, Edmunds 2013). Porites is both the most abundant genus in the quasi-stationary distribution (Fig. 5), and it has shown the strongest increasing trend in coral cover (Fig. 6). In contrast to Porites, Agaricia appears susceptible to hurricanes and seawater warming (Fig. 6). This finding corroborates suggestions that Agaricia will be rarer in future communities because of its high susceptibility to thermal stress (Aronson et al. 2000, Smith et al. 2013). All other common genera at the RS habitat appear relatively robust to hurricanes and seawater warming, although the low mean cover of some genera (notably Montastrea) likely compromised statistical power to detect modest sensitivities here. Together, the taxonomic results reinforce the notion that responses of scleractinians to environmental conditions will vary predictably among coral genera, suggesting that future reef communities will consist of assemblages with a greater proportion of "winning" taxa and fewer "losing" taxa (Loya et al. 2001, Darling et al. 2012, Edmunds et al. 2014).

What do the results of this analysis portend for coral communities in St. John in the near future, if environments become even harsher? Our analysis shows that the stability of these communities is nuanced, and suggests heterogeneous responses to environmental change even within $\sim 4 \mathrm{~km}$ of shore. At Tektite and Yawzi Point, lower (scaled) annual variation in coral cover is coupled with slower recovery from disturbance. This finding is consistent with the expectation of slow population dynamics for long-lived, sporadically recruiting taxa such as Orbicella spp. vs. more rapid dynamics for the short-lived, rapidly recruiting corals (i.e., "weedy" taxa) of the RS habitat. However, sensitivities suggest that corals at Yawzi Point (already considerably more scarce than 25 years ago) are vulnerable to additional coral loss if hurricanes become more frequent or seas become warmer. Trend statistics also suggest that corals at Tektite and Yawzi Point have experienced chronic losses 
that cannot be attributed to the immediate impacts of hurricanes and warming. Without a mechanistic explanation for these trends, it is difficult to suggest whether they may continue in future years. However, the slow dynamics already revealed in these habitats suggest that additional coral losses will require multiple years of less disturbed conditions if these communities are to recover their abundance of 25 years ago.

Coral at the RS habitats, on the other hand, may exhibit highly variable cover across time and space, but always cover only a small fraction of the benthos. These coral communities quickly recover from disturbance, and with the possible exception of Agaricia spp., appear relatively robust to further change by environmental stressors. The quick recovery and relative insensitivity to environmental stress of the coral communities in the RS habitat suggest that, while some coral genera (e.g., Porites) may increase in abundance and others (Agaricia) may decline in coming years, the aggregate coral cover and benthic community of the near future is likely to be fairly similar to that of the recent past. This stasis suggests stability, but by and large it is a stability borne of coral rarity. That is, one interpretation of the present results is that the stability of the RS habitat is a consequence of their low coral cover and the absence of larger, longer-lived, more structurally complex coral colonies like those of Orbicella. Thus, while the stability of the RS habitat is a real property of these communities, it could also indicate a degraded ecosystem with little left to lose.

We close with a methodological comment. MAR models require time series of considerable duration to yield precise and unbiased estimates of stability. In the present analysis, two decades of data provide enough statistical power to identify the largest effects. However, smaller environmental effects (e.g., sensitivity of coral to sea temperature at Tektite), and more subtle differences in stability between ecosystems must be viewed with caution when they fail to meet conventional standards of statistical significance, or (in the case of spectral radii) may be estimated with considerable bias. As a practical matter, the broader adoption of MAR models to estimate stability from monitoring data would be facilitated by a deeper understanding of the smallsample statistical properties of these models, including power and bias. Evaluating the properties of statistical estimators in MAR models will be complicated, and will depend on a multitude of factors, including the number of taxa, the generation time of those taxa, the number of environmental covariates, and pre-existing biological knowledge that can be used to structure the matrices $\mathbf{B}$ or C. Yet, even a rough understanding of the relationship between data duration and the properties of its statistical estimators would be a welcome advance, and could inform both the design and continuation of monitoring studies, and strategic decisions at the time of analysis.

\section{ACKNOWLEDGMENTS}

The data analyzed in this article would not have been collected without the support of many field assistants, dedicated shore-side staff, and funding from numerous sources, including most recently the NSF LTREB program (DEB 03-43570, 0841441, and 13-50146). This project was catalyzed by the NCEAS (NSF grant EF-0553768) working group "Tropical coral reefs of the future." We thank M. Spencer, J. F. Bruno, two anonymous reviewers, and Subject Matter Editor E. Holmes for thoughtful discussion and comments about the manuscript.

\section{Literature Cited}

Arnold, S. N., R. S. Steneck, and P. J. Mumby. 2010. Running the gauntlet: inhibitory effects of algal turfs on the processes of coral recruitment. Marine Ecology Progress Series 414:91105 .

Aronson, R. B., W. F. Precht, I. G. Macintyre, and T. J. T. Murdoch. 2000. Ecosystems: coral bleach-out in Belize. Nature 405:36.

Bellard, C., C. Bertelsmeier, P. Leadley, W. Thuiller, and F. Courchamp. 2012. Impacts of climate change on the future of biodiversity. Ecology Letters 15:365-377.

Burman, S. G., R. B. Aronson, and R. van Woesik. 2012. Biotic homogenization of coral assemblages along the Florida reef tract. Marine Ecology Progress Series 467:89-96.

Connell, J. H. 1978. Diversity in tropical rain forests and coral reefs. Science 199:1302-1310.

Cooper, J. K., M. Spencer, and J. F. Bruno. 2015. Stochastic dynamics of a warmer Great Barrier Reef. Ecology 96:18021811.

Darling, E. S., L. Alvarez-Filip, T. A. Oliver, T. R. McClanahan, and I. M. Côté. 2012. Evaluating life-history strategies of reef corals from species traits. Ecology Letters 15:13781386.

Donner, S. D. 2009. Coping with commitment: projected thermal stress on coral reefs under different future scenarios. PLoS ONE 4:e5712.

Edmunds, P. J. 2002. Long-term dynamics of coral reefs in St. John, US Virgin Islands. Coral Reefs 21:357-367.

Edmunds, P. J. 2013. Decadal-scale changes in the community structure of coral reefs of St. John, US Virgin Islands. Marine Ecology Progress Series 489:107-123.

Edmunds, P. J., et al. 2014. Persistence and change in community composition of reef corals through present, past, and future climates. PLoS ONE 9(10):e107525.

Edmunds, P. J., and S. C. Gray. 2014. The effects of storms, heavy rain, and sedimentation on the shallow coral reefs of St. John, US Virgin Islands. Hydrobiologia 734:1-16.

Edmunds, P. J., and J. D. Witman. 1991. Effect of Hurricane Hugo on the primary framework of a reef along the south shore of St. John, US Virgin Islands. Marine Ecology Progress Series 78:201-204.

Efron, B., and R. J. Tibshirani. 1994. An introduction to the bootstrap. CRC Press, Boca Raton, Florida, USA.

Egozcue, J. J., and V. Pawlowsky-Glahn. 2011. Basic concepts and procedures. Pages 12-28 in V. Pawlowsky-Glahn and A. Buccianti, editors. Compositional data analysis: theory and applications. John Wiley and Sons, New York, New York, USA.

Gardner, T. A., I. M. Côté, J. A. Gill, A. Grant, and A. R. Watkinson. 2003. Long-term region-wide declines in Caribbean corals. Science 301:958-960.

Gledhill, D. K., R. Wanninkhof, F. J. Millero, and M. Eakin. 2008. Ocean acidification of the greater Caribbean region 1996-2006. Journal of Geophysical Research 113. http://dx. doi.org/10.1029/2007JC004629

Gosz, J. R., R. B. Waide, and J. J. Magnuson. 2010. Twentyeight years of the US-LTER program: experience, results, and research questions. Pages 59-74 in F. Müller, C. 
Baessler, H. Schubert, and S. Klotz, editors. Long-term ecological research. Springer, Dordrecht, The Netherlands.

Hampton, S. E., E. E. Holmes, L. P. Scheef, M. D. Scheuerell, S. L. Katz, D. E. Pendleton, and E. J. Ward. 2013. Quantifying effects of abiotic and biotic drivers on community dynamics with multivariate autoregressive (MAR) models. Ecology 94:2663-2669.

Hernández-Pacheco, R., E. A. Hernández-Delgado, and A. M. Sabat. 2011. Demographics of bleaching in a major Caribbean reef-building coral: Montastraea annularis. Ecosphere 2:art9.

Hoegh-Guldberg, O. 1999. Climate change, coral bleaching and the future of the world's coral reefs. Marine and Freshwater Research 50:839-866.

Hoegh-Guldberg, O., et al. 2007. Coral reefs under rapid climate change and ocean acidification. Science 318:17371742.

Holling, C. S. 1973. Resilience and stability of ecological systems. Annual Review of Ecology and Systematics 4:1-23.

Ives, A. R., and S. R. Carpenter. 2007. Stability and diversity of ecosystems. Science 317:58-62.

Ives, A. R., and V. Dakos. 2012. Detecting dynamical changes in nonlinear time series using locally linear state-space models. Ecosphere 3:art58.

Ives, A. R., B. D. Dennis, K. L. Cottingham, and S. R. Carpenter. 2003. Estimating community stability and ecological interactions from time-series data. Ecological Monographs 73:301-330.

Jackson, J. B. C., M. K. Donovan, K. L. Cramer, and V. V. Lam. 2014. Status and trends of Caribbean coral reefs. Global Coral Reef Monitoring Network, IUCN, Gland, Switzerland.

Knowlton, N., J. C. Lang, and B. D. Keller. 1990. Case study of natural population collapse: post-hurricane predation on Jamaican staghorn corals. Smithsonian Contributions to the Marine Sciences Number 31:1-25.

Kroeker, K. J., R. L. Kordas, R. Crim, I. E. Hendriks, L. Ramajo, G. S. Singh, C. M. Duarte, and J.-P. Gattuso. 2013. Impacts of ocean acidification on marine organisms: quantifying sensitivities and interaction with warming. Global Change Biology 19:1884-1896.

Lesser, M. P. 2011. Coral bleaching: causes and mechanisms. Pages 405-419 in Coral reefs: an ecosystem in transition. Springer, New York, New York, USA.

Levitan, D. R., P. J. Edmunds, and K. E. Levitan. 2014. What makes a species common? No evidence of density-dependent recruitment or mortality of the sea urchin Diadema antillarum after the 1983-1984 mass mortality. Oecologia 175:117-128.

Lindenmayer, D. B., and G. E. Likens. 2010. The science and application of ecological monitoring. Biological Conservation 143:1317-1328.

Logan, C. A., J. P. Dunne, C. M. Eakin, and S. D. Donner. 2014. Incorporating adaptive responses into future projections of coral bleaching. Global Change Biology 20:125-139.

Lovett, G. M., D. A. Burns, C. T. Driscoll, J. C. Jenkins, M. J. Mitchell, L. Rustad, J. B. Shanley, G. E. Likens, and R. Haeuber. 2007. Who needs environmental monitoring? Frontiers in Ecology and the Environment 5:253-260.

Loya, Y., K. Sakai, K. Yamazato, Y. Nakano, H. Sambali, and R. Van Woesik. 2001. Coral bleaching: the winners and the losers. Ecology Letters 4:122-131.
May, R. M. 1974. Stability and complexity in model ecosystems. Princeton University Press, Princeton, New Jersey, USA.

McClanahan, T. R., M. Ateweberhan, C. A. Muhando, J. Maina, and M. S. Mohammed. 2007. Effects of climate and seawater temperature variation on coral bleaching and mortality. Ecological Monographs 77:503-525.

Miller, J., E. Muller, C. Rogers, R. Waara, A. Atkinson, K. R. T. Whelan, M. Patterson, and B. Witcher. 2009. Coral disease following massive bleaching in 2005 causes $60 \%$ decline in coral cover on reefs in the US Virgin Islands. Coral Reefs 28:925-937.

Mumby, P. J., A. Hastings, and H. J. Edwards. 2007. Thresholds and the resilience of Caribbean coral reefs. Nature 450:98-101.

Nyström, M., and C. Folke. 2001. Spatial resilience of coral reefs. Ecosystems 4:406-417.

Parmesan, C. 2006. Ecological and evolutionary responses to recent climate change. Annual Review of Ecology, Evolution, and Systematics 37:637-669.

Parmesan, C., M. T. Burrows, C. M. Duarte, E. S. Poloczanska, A. J. Richardson, D. S. Schoeman, and M. C. Singer. 2013. Beyond climate change attribution in conservation and ecological research. Ecology Letters 16:58-71.

Rogers, C. S. 1993. Hurricanes and coral reefs: the intermediate disturbance hypothesis revisited. Coral Reefs 12:127-137.

Rogers, C. S., et al. 2008. Ecology of coral reefs in the US Virgin Islands. Pages 303-373 in B. M. Riegl and R. E. Dodge, editors. Coral Reefs of the USA. Springer, Dordrecht, The Netherlands.

Rosenberg, E., and Y. Loya. 2004. Coral health and disease. Springer, Dordrecht, The Netherlands.

Sandin, S. A., et al. 2008. Baselines and degradation of coral reefs in the northern Line Islands. PLoS ONE 3:e1548.

Scheffer, M., J. Bascompte, W. A. Brock, V. Brovkin, S. R. Carpenter, V. Dakos, H. Held, E. H. Van Nes, M. Rietkerk, and G. Sugihara. 2009. Early-warning signals for critical transitions. Nature 461:53-59.

Schutte, V. G. W., E. R. Selig, and J. F. Bruno. 2010. Regional spatio-temporal trends in Caribbean coral reef benthic communities. Marine Ecology Progress Series 402: $115-122$.

Smith, T. B., M. E. Brandt, J. M. Calnan, R. S. Nemeth, J. Blondeau, E. Kadison, M. Taylor, and P. Rothenberger. 2013. Convergent mortality responses of Caribbean coral species to seawater warming. Ecosphere 4:art87.

Stocker, T. F., D. Qin, G. K. Plattner, M. Tignor, S. K. Allen, J. Boschung, A. Nauels, Y. Xia, V. Bex, and P. M. Midgley. 2013. Climate change 2013: the physical science basis. Contribution of Working Group I to the Fifth Assessment Report of the Intergovernmental Panel on Climate Change. Cambridge University Press, Cambridge, UK.

Van Hooidonk, R., J. A. Maynard, and S. Planes. 2013. Temporary refugia for coral reefs in a warming world. Nature Climate Change 3:508-511.

Walther, G.-R., E. Post, P. Convey, A. Menzel, C. Parmesan, T. J. C. Beebee, J.-M. Fromentin, O. Hoegh-Guldberg, and F. Bairlein. 2002. Ecological responses to recent climate change. Nature 416:389-395.

\section{Supplemental Material}

\section{Ecological Archives}

Appendices A-E are available online: http://dx.doi.org/10.1890/14-0941.1.sm 